\title{
ÚTERO SEPTADO, DUPLICACIÓN DEL CUELLO Y SEPTO VAGINAL: INFORME DE UNA RARA MALFORMACIÓN
}

\author{
Septate uterus, cervical duplication and vaginal \\ septum: a report of an uncommon malformation
}

\section{Septo uterino, duplicação cervical e septo vaginal:} relato de uma imalformação incomuım

Mariangela Badalotti*, Adriana Arent **, Vicente Monteggia***, Julia Machado****, Rafaella Petracco*****, Alvaro Petracco******

Recibido: mayo 19/2005 - Revisado: julio 26/2005 - Aceptado: agosto 12/2005

\section{RESUMEN}

Objetivo: describir una rara alteración de la fusión de los canales de Müller.

Diseño: presentación de caso

Reporte del caso: una paciente asiste a la consulta con un examen de ultrasonido en cuya descripción se plantea la sospecha de útero septado. El examen ginecológico reveló un septo vaginal longitudinal, que llegaba hasta la región del himen y la presencia de dos cuellos. La ecografía pélvica tridimensional mostró duplicación del cuello, útero septado que comprometía desde el istmo hasta la cavidad uterina

\footnotetext{
* $\quad$ Médica Ginecologista. Professora Mestre da Faculdade de Medicina PUCRS. Chefe do Serviço de Ginecologia do HSL/PUCRS.

Diretora do Fertilitat - Centro de Medicina Reprodutiva.

** Médica Ginecologista. Preceptora Mestre do Serviço de Ginecologia do HSL/PUCRS. Membro do Fertilitat - Centro de Medicina Reprodutiva.

*** Médico Ginecologista especialista em ultra-sonografia

**** Médica Residente do Serviço de Ginecologia e Obstetrícia do HSL PUCRS

****** Acadêmica da Faculdade de Medicina da ULBRA.

******* Médico Ginecologista. Professor da Faculdade de Medicina PUCRS Diretor do Fertilitat - Centro de Medicina Reprodutiva.

Trabajo realizado nel Fertilitat - Centro de Medicina Reprodutiva. Porto Alegre, Brasil.

Dirección para correspondencia: Adriana Arent - Quintino Bocaiúva, 1617/301 - Porto Alegre, RS - Brasil - CEP:90440-051

Correo electrónico: adriarent@ig.com.br fertilitat@fertilitat.com.br
}

pero sin división del cuerpo uterino, compatible con útero septado y cuello doble.

Conclusión: este caso representa una rara malformación no incluida dentro de la clasificación habitual de las malformaciones Müllerianas y que no tiene explicación embriogénica, que se apoya en la teoría de la fusión unidireccional de los canales de Müller. La ecografía tridimensional, es un examen simple y de bajo costo y demostró ser una buena opción para el diagnóstico definitivo de esta malformación, por lo que debe ser considerada dentro del arsenal de exámenes complementarios.

Palabras clave: útero, cérvix uterino, vaginal, ductos müllerianos.

\section{SUMMARY}

Objective: to describe a case of an unusual müllerian anomaly.

Design: case report.

Case report: a 34 years old white nulligravida presented with complains of suspected uterine septum observed during a routine ultrasonographic examination. Gynecological examination revealed a longitudinal vaginal septum which arrived in hymeneal region and two uterine cervixes. Three 
dimensional pelvic ultrasonography showed cervix duplication, uterine septum from isthmus to endometrial cavity and absence of uterine body division, compatible with complete uterine septum and true dual cervices.

Conclusion: this case represents a rare malformation that is not included in usual classification of müllerian malformations and is not explained by the traditional embryologic vision that supports the unidirectional müllerian fusion. The three-dimensional ultrasonography, a non-invasive and lower cost exam, is a diagnostic option that must be considered in diagnosis arsenal of müllerian malformations.

Key words: uterus, cervix uteri, vaginal, Müllerian ducts.

\section{INTRODUÇÃO}

No desenvolvimento mülleriano normal parece existir uma progressão ordenada do desenvolvimento embrionário do útero, trompas uterinas e vagina. Uma alteração deste processo resulta nas anomalias uterinas congênitas. As malformações müllerianas representam um conjunto de anomalias estruturais que podem se originar da falha de fusão dos dutos müllerianos na linha média, na falha na conexão com seio urogenital, ou na falha quanto a criar uma luz apropriada na porção superior da vagina e útero, por absorção das células da vagina central e do septo entre os dutos müllerianos fundidos. ${ }^{1}$ Porém a causa definitiva destas anomalias não está claramente elucidada. Estimase que as anomalias müllerianas acometem, aproximadamente, 1 a $6 \%$ das mulheres, porém a incidência exata destas malformações é incerta, pois a maioria dos dados são resultados de estudos de complicações obstétricas ou infertilidade. ${ }^{1,2}$ Varias classificações foram propostas para as anomalias uterinas congênitas, baseadas no entendimento da embriologia do sistema mulleriano, sendo a mais utilizada a posposta por Buttram e Gibbons, ${ }^{3}$ posteriormente modificada pela American Fertility Society. ${ }^{4}$

O septo uterino é a malformação uterina congênita mais comum, porém apenas 14\% das pacientes com septo uterino apresentam um septo completo com envolvimento cervical. Ainda mais rara é a ocorrência de duplicação cervical verdadeira associada a septo uterino completo e septo vaginal longitudinal.

Esta é uma malformação que, além de rara, não se enquadra na teoria clássica de desenvolvimento embrionário e na classificação da ASRM, estando descritos não mais de 23 casos na literatura. ${ }^{5,6}$ Apresentamos um novo caso desta anomalia, cujo diagnóstico foi realizado com ecografia pélvica tridimensional, no intuito de alertar a possibilidade deste tipo de malformação.

\section{RELATO DO CASO}

Paciente de 34 anos, branca, nuligesta, veio à avaliação por suspeita de septo uterino em exame ecográfico bidimensional. Referia desenvolvimento puberal normal com menarca aos 12 anos, ciclos menstruais regulares, com quatro dias de fluxo normal e dismenorréa leve. Negava história materna de exposição a dietilbestrol e não havia conhecimento de malformações de sistema urinário.

Ao exame ginecológico, apresentava genitália externa normal e septo vaginal longitudinal, que se estendia até à região himenial, e presença de dois colos uterinos. No toque vaginal palpava-se os dois colos, o útero anteversofletido e não havia massas pélvicas. A ecografia transvaginal demonstrava duas cavidades uterinas com distância intercornual

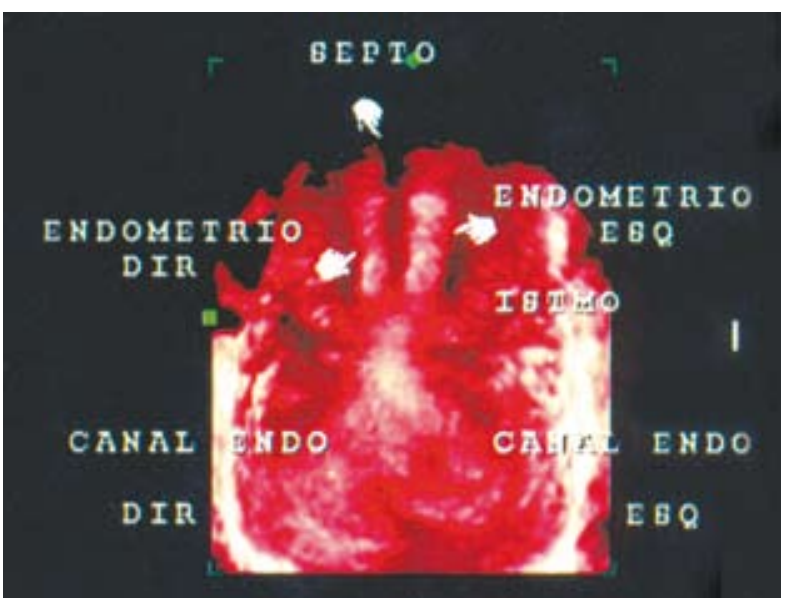

Figura 1. Septo uterino e duplicação de canal endocervical. 
normal. Para elucidação diagnóstica, foi solicitada ecografia pélvica tridimensional que evidenciou duplicação cervical (figura 1), septo uterino que se estendia do istmo ao final da cavidade endometrial e ausência de divisão do corpo uterino (figura 2), compatível com útero septado completo e duplicação cervical verdadeira.

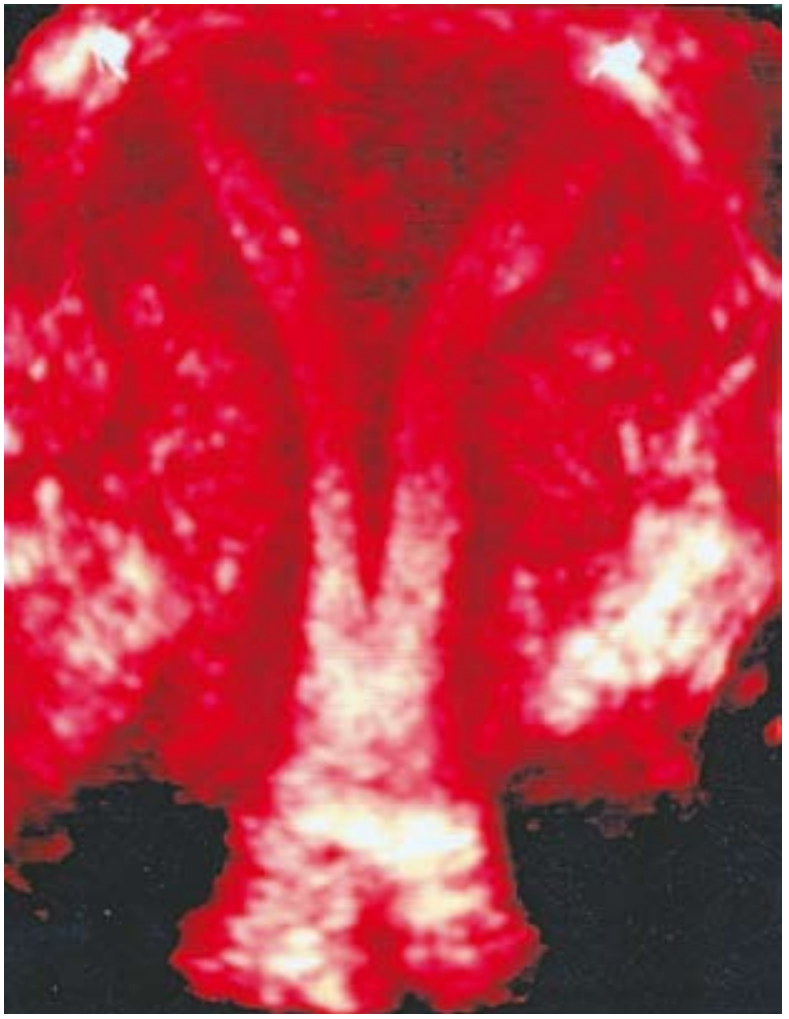

Figura 2. Ausência de divisão de corpo uterino.

\section{Local}

Fertilitat - Centro de Medicina Reprodutiva. Centro privado de atendimento terciário

Acreditado pela Red Latinoamericana de Reproducción Asistida.

Porto Alegre, RS, Brasil.

\section{Ética}

Todas pacientes da clínica Fertilitat assinam termo de consentimento informado antes da realização de tratamentos, disponibilizando a utilização de seus dados para fins científicos; sendo este procedimento aprovado pelo Comitê de Ética em Pesquisa da Pontifícia Universidade Católica do Rio Grande do Sul. A confidencialidade da paciente foi preservada neste relato, sendo apresentados apenas dados da história médica sem a identificação da paciente.

\section{DISCUSSÃO}

Várias tentativas foram feitas durante anos para estabelecer uma classificação compreensiva das anomalias dos ductos müllerianos. ${ }^{3,4,7}$ Estas classificações são compatíveis com as hipóteses sobre o desenvolvimento embriológico normal do sistema mülleriano assim como a formação das anomalias müllerianas. A teoria clássica, que sustenta que a fusão mülleriana ocorra de maneira unidirecional, no sentido caudal-cefálico, foi detalhada por Crosby e Hill em 1962. ${ }^{7}$

Neste caso descrevemos a duplicação cervical associada a septo vaginal e uterino com presença de fundo uterino normal. Esta rara anomalia foi primeiramente descrita em $1994 .^{2}$ Desde então outros relatos foram feitos, alguns isolados e outros em séries, totalizando 23 casos até o momento. ${ }^{5,6,8,9}$ Após estas publicações, a clássica teoria do desenvolvimento unidirecional (caudal-cranial) dos ductos müllerianos foi questionada e foi proposta uma teoria alternativa bidirecional, que sustenta que a fusão e a reabsorção iniciem no istmo e procedam, simultaneamente, em ambas direções cranial e caudal. ${ }^{10}$ Concordando com esta teoria, McBean et al sugerem que o útero septado com duplicação cervical e septo vaginal longitudinal possa ser explicado pela falha na fusão de dois ductos müllerianos distais, com desenvolvimento normal do resto do útero, causando a duplicação cervical, com a presença de um fundo uterino único. Já os septos uterino e vaginal são conseqüência da falha completa na reabsorção septal ou canalização. A teoria clássica da fusão unidirecional não consegue explicar adequadamente esta rara anomalia descrita.

Casos de septo uterino completo associado a septo vaginal podem ser facilmente confundidos com útero didelfo. Entretanto, o manejo destas 
duas anomalias müllerianas é diverso, sendo a correção cirúrgica usualmente necessária nos casos de septo uterino completo. No que tange aos propósitos diagnósticos dos relatos anteriores, Wait et $\mathrm{al}^{11}$ enfatizaram a utilidade da histeroscopia laparoscopicamente assistida, enquanto que os estudos mais recentes propõem a ressonância nuclear magnética (RNM) para o diagnóstico definitivo e não invasivo desta rara anomalia. ${ }^{6,9} \mathrm{O}$ presente caso é interessante porque demonstra o valor da ecografia pélvica tridimensional como um método de imagem diagnóstico definitivo para avaliação da anatomia uterina e diagnóstico das anomalias müllerianas. A ultrassonografia tridimensional é um exame menos invasivo e menos caro que a histeroscopia/laparoscopia. Assim como a RNM pode ser realizada ambulatorialmente, porém tem menor custo. A possibilidade de visualização da cavidade uterina e do miométrio com a ecografia tridimensional facilita o diagnóstico das anomalias uterinas, proporcionando a diferenciação entre septo uterino e útero bicorno e permitindo ainda o planejamento cirúgico. ${ }^{12,13}$ No caso descrito, além da avaliação do corpo uterino (figura 2), foi possível evidenciar a presença dos dois colos uterinos (figura 1).

Este é, segundo nosso conhecimento, o 240 caso desta rara malformação, cujo diagnóstico é geralmente retardado até a idade reprodutiva, devido ao fato de não causar problemas durante a puberdade. O crescente número de casos descritos desta anomalia desde o primeiro relato em 1994 deve ser resultado do aumento de acesso e acurácia dos métodos de imagem como a RNM e ecografia tridimensional.

\section{CONCLUSÃO}

Duplicação cervical e septo vaginal associados a septo uterino é um tipo incomum de malformação mülleriana. Este caso representa uma rara malformação que não está incluída na classificação usual das anomalias müllerianas e é melhor explicada pela teoria embriológica que suporta que a fusão mülleriana se procede bidirecionalmente. O crescente número desta malformação indica a presença de um grupo adicional de anomalias müllerianas que ainda não se encontra nas classificações utilizadas. Como ainda não existe consenso quanto ao impacto desta anomalia sobre a fertilidade, estas pacientes devem ser seguidas no intuito de se estabelecer o melhor plano terapêutico a ser seguido. A ultrassonografia tridimensional, exame não invasivo e de baixo custo, é uma opção diagnóstica que deve ser considerada no arsenal semiótico das mal-formações müllerianas.

\section{REFERÊNCIAS}

1. Acien P, Acien M, Sanchez-Ferrer M. Complex malformations of the female genital tract. New types and revision of classification. Hum Reprod 2004;19:2377-84.

2. McBean JH, Brumsted JR. Septate uterus with cervical duplication: a rare malformation. Fertil Steril 1994;62:415-7.

3. Buttram VC, Gibbons WE. Mullerian anomalies: a proposed classification (an analysis of 144 cases). Fertil Steril 1979;32:40-6.

4. The American Fertility Society. The AFS classifications of adnexal adhesions, distal tubal occlusion tubal occlusion secondary to tubal ligation, tubal pregnancies, Müllerian anomalies and intrauterine adhesions. Fertil Steril 1988;49:944-55.

5. Saygili-Yilmaz ES, Erman-Akar M, Bayar D, Yuksel B, Yilmaz Z. Septate uterus with a double cervix and longitudinal vaginal septum. J Reprod Med 2004;49:833-6.

6. Chang AS, Siegel CL, Moley KH, Ratts VS, Odem RR Septate uterus with cervical duplication and longitudinal vaginal septum: a report of five new cases. Fertil Steril 2004;81:1133-6.

7. Crosby WM, Hill EC. Embriology of the Mullerian duct system. Review of present-day theory. Obstet Gynecol 1962;20:507-15.

8. Balasch J, Moreno E, Martinez-Roman S, Molini JL, Torne A, Sanchez-Martin F, et al. Septate uterus with cervical duplication: a report of three new cases. Eur J Obstet Gynecol Reprod Biol 1996;65:241-3.

9. Hundley AF, Fielding JR, Hoyte L. Double cervix and vagina with septate uterus: an uncommon mullerian malformation. Obstet Gynecol 2001;98:982-5. 
10. Muller P, Musset R, Netter A, Solal R, Vinourd JC, Gillel JY. Etat du haut appareil urinaire chez les porteuses de malformations uterines: etude de 133 observations. Presse Med 1967;75:1331-6.

11. Wai CY, Zekam N, Sanz LE. Septate uterus with double cervix and longitudinal vaginal septum. J Reprod Med 2001;46:613-7.
12. Kupesic S, Kurjak A. Diagnosis and treatment outcome of the septate uterus. Croat Med J 1998; 39:185-90.

13. Wu MH, Hsu CC, Huang KE. Detection of congenital mullerian duct anomalies using three-dimensional ultrasound. J Clin Ultrasound 1997;25:487-92.

Conflicto de intereses: ninguno declarado. 